\title{
Protecting Canada's children from the consequences of the fourth wave of the COVID-19 pandemic
}

\author{
Stephen B. Freedman MDCM MSc, James D. Kellner MD MSc
}

Cite as: CMAJ 2021 September 27;193:E1500-2. doi: 10.1503/cmaj.211513

See related article at www.cmaj.ca/lookup/doi/10.1503/cmaj.210053

$\mathbf{U}$ nderstanding and responding to the impact of SARSCoV-2 infections on children is a high priority as the fourth wave of the COVID-19 pandemic sweeps across Canada. Although highly effective vaccines are available to prevent infections in people 12 years of age and older, none are yet approved for use in younger children, the timelines to approval remain uncertain and uptake is unpredictable. In this context, a related study using data ${ }^{1}$ from the Canadian Paediatric Surveillance Program (CPSP) provides information on the frequency and characteristics of children admitted to hospital with COVID19 , a key indicator of the pediatric burden of disease. However, public policy must integrate other considerations including the impact of new SARS-CoV-2 variants (e.g., delta variant), incidence of multisystem inflammatory syndrome in children (MIS-C) and post-COVID conditions, and availability and uptake of COVID-19 vaccines.

The related study ${ }^{1}$ clearly shows that children were relatively spared during the first 2 waves of the pandemic. Drouin and colleagues note that 308 cases of children admitted to hospital with SARS-CoV-2 infections were reported to the Public Health Agency of Canada by Dec. 31, 2020. Through the CPSP reporting system, the authors analyzed records for 264 children who were admitted to hospital with acute SARS-CoV- 2 infection for which detailed chart review information was submitted. Of these cases, just 150 (57\%) were categorized as admitted to hospital because of COVID-19. This low number reflects the mild nature of SARS-CoV-2 infection in children and that public health measures effectively prevented widespread disease in children.

An analysis of data from electronic health records performed in September 2020, which included more than 135000 people in the United States who were younger than 25 years of age, reported that $7 \%$ of those infected with SARS-CoV-2 were admitted to hospital, with $28 \%$ being admitted to an intensive care unit (ICU). ${ }^{2}$ Although the related study ${ }^{1}$ does not describe the proportion of children

\section{Key points}

- Few children were admitted to hospital in Canada because of COVID-19 during the early waves of the pandemic; however, severe illness was common among chidren admitted to hospital.

- Delayed ramifications from SARS-CoV-2 infections (e.g., multisystem inflammatory syndrome and post-COVID conditions) may have a greater impact on the health of children than acute illness, and the burden of these should be considered and studied, particularly in the context of the delta variant.

- Because children under 12 years of age are not yet able to receive COVID-19 vaccines in Canada, it is important to ensure vaccination of all those who are eligible and to maintain other mitigation measures, such as masking and improved ventilation, to protect children as a new school year begins.

with SARS-CoV-2 infection who required hospital admission, the authors do report that among those children who were admitted to hospital with COVID-19, 21\% were admitted to an ICU and $6 \%$ required mechanical ventilation. According to the latest Canadian data (as of Aug. 29, 2021), the overall proportion of children with SARS-CoV-2 infection who required hospital admission is low (0.5\%); however, $11 \%$ required ICU admission. ${ }^{3}$ These relative proportions of children in hospital with severe disease are concerning. When compared with other pediatric respiratory illnesses, these proportions of admitted children requiring ICU care exceed those reported for children with bronchiolitis, ${ }^{4}$ and seasonal and pandemic H1N1 influenza. ${ }^{5}$

The cases reported in the related study ${ }^{1}$ occurred before emergence of the SARS-CoV-2 delta variant. This new variant requires that we reconsider risk. The delta variant is more transmissible than previous strains, and emerging evidence describes higher morbidity at all ages, including in children. A 
cohort study that included all patients (30\% were younger than $20 \mathrm{yr}$ of age) with COVID-19 in England in the spring of 2021 reported that those with infections caused by the delta variant were more than twice as likely to be admitted to hospital than those with alpha variant infections, particularly those who were unvaccinated. ${ }^{6}$ This raises concerns about the impact the delta variant may have in isolation, or when combined with other respiratory illnesses, on children during the fall-winter of 2021-2022.

SARS-CoV-2 infection may also lead to the postinfectious complication MIS-C. The incidence of MIS-C is estimated to be $316 / 1000000$ SARS-CoV-2 infections (1/3165 children with SARS-CoV-2 infection). ${ }^{7}$ Children with MIS-C are usually very ill, with nearly $75 \%$ requiring ICU admission and $2 \%$ dying. ${ }^{8}$ It remains unknown whether the current wave, driven by the delta variant, insufficient levels of vaccination in the overall population with no vaccination of children under 12 years and reduced public health measures, will lead to a surge of cases of MIS-C. However, past waves have shown recurrently that MIS-C peaks 4-6 weeks after surges in SARS-CoV-2 infections, thus a similar increase in cases should be anticipated to follow the rise in pediatric cases during the fourth wave of the pandemic in Canada.

The constellation of post-COVID conditions must also be considered. Although well characterized in adults who have had severe acute COVID-19, lasting effects are increasingly reported in people after mild SARS-CoV-2 infections. The prevalence of post-COVID conditions appears lower in children than in adults, although definitive studies are lacking, but there is a consistent trend toward a greater proportion of children with SARS-CoV-2 infection, relative to control (children with negative test results), reporting ongoing symptoms several months after infection. ${ }^{9,10}$ Nonetheless, evidence that post-COVID conditions do occur in children is mounting, and they must be considered when quantifying the burden of SARS-CoV-2 infection in children.

Taken together, these concerns highlight the importance of ongoing efforts to mitigate SARS-CoV-2 infections in children. Canada is at a crucial point in time in the COVID-19 pandemic, the fourth wave is rapidly gaining momentum and a new school year is starting. Most children will be attending school in person and, because children under 12 years of age are not yet eligible for vaccination, they are a particularly vulnerable group. It is imperative that school shutdowns be avoided to avert their impact on children's mental health and their academic progress. At present, however, there is considerable variation in preventive measures being taken within schools and other settings where children gather across Canada. Airborne spread of SARS-CoV-2 is a particular concern in school settings, as locations with inadequate ventilation, where a person who is infected may be present for an extended time period, can lead to the accumulation of a sufficient quantity of airborne particles to permit the transmission of infection. A paucity of robust data to support use of masks was available in the early months of the pandemic, but now there is evidence that mask wearing in the community effectively reduces the spread of SARS-CoV-2 and, to a lesser extent, protects the wearer. ${ }^{11}$ Although wearing masks may be uncomfortable, they do not substantially impact children's ability to infer and respond to emotions, ${ }^{12}$ and they represent an important safety measure that should be implemented to protect children.

Although, as shown by the authors of the related study, ${ }^{1}$ the consequences of acute COVID-19 in children were limited in the early phases of the pandemic, the direct and indirect impacts of SARS-CoV-2 infections in children must be considered when determining public health policies. These deliberations must integrate the short- and long-term impacts that public policy may have on the physical, mental and social well-being of children. While the light is visible at the end of the tunnel, children in Canada must continue to be protected as they may be the last ones to get there.

\section{References}

1. Drouin O, Moore Hepburn C, Farrar DS, et al. Characteristics of children admitted to hospital with acute SARS-CoV-2 infection in Canada in 2020. CMAJ 2021;193:EX-X.

2. Bailey LC, Razzaghi H, Burrows EK, et al. Assessment of 135794 pediatric patients tested for severe acute respiratory syndrome coronavirus 2 across the United States. JAMA Pediatr 2021;175:176-84.

3. COVID-19 daily epidemiology update. Ottawa: Government of Canada; 2021. Available: https://health-infobase.canada.ca/covid-19/epidemiological-summary -covid-19-cases.html (accessed 2021 Aug. 29).

4. Oakley E, Chong V, Borland M, et al. Intensive care unit admissions and ventilation support in infants with bronchiolitis. Emerg Med Australas 2017;29: 421-8.

5. Tran D, Vaudry W, Moore DL, et al. Comparison of children hospitalized with seasonal versus pandemic influenza A, 2004-2009. Pediatrics 2012;130: 397-406.

6. Twohig KA, Nyberg T, Zaidi A, et al. Hospital admission and emergency care attendance risk for SARS-CoV-2 delta (B.1.617.2) compared with alpha (B.1.1.7) variants of concern: a cohort study. Lancet Infect Dis 2021;S1473 -3099:00475-8.

7. Payne AB, Gilani Z, Godfred-Cato S, et al. Incidence of multisystem inflammatory syndrome in children among US persons infected with SARS-CoV-2. JAMA Netw Open 2021;4:e2116420.

8. Feldstein LR, Tenforde MW, Friedman KG, et al. Characteristics and outcomes of US children and adolescents with multisystem inflammatory syndrome in children (MIS-C) compared with severe acute COVID-19. JAMA 2021;325: 1074-87.

9. Molteni E, Sudre CH, Canas LS, et al. Illness duration and symptom profile in symptomatic UK school-aged children tested for SARS-CoV-2. Lancet Child Adolesc Health 2021 Aug. 3 [Epub ahead of print]. doi: https://doi.org/10.1016/ S2352-4642(21)00198-X.

10. Stephenson T, Pinto Pereira S, Shafran R, et al.; CLoCK Consortium. Long COVID - the physical and mental health of children and non-hospitalised young people 3 months after SARS-CoV-2 infection; a national matched cohort study (The CLoCk study). In Review [preprint] 2021 Aug. 10. doi: 10.21203/ rs.3.rs-798316/v1.

11. Johansson MA, Quandelacy TM, Kada S, et al. SARS-CoV-2 transmission from people without COVID-19 symptoms. JAMA Netw Open 2021;4: e2035057-e.

12. Ruba AL, Pollak SD. Children's emotion inferences from masked faces: implications for social interactions during COVID-19. PLOS One 2020;15: e0243708. 
Competing interests: Stephen Freedman's research is supported by the Alberta Children's Hospital Foundation Professorship in Child Health and Wellness. James Kellner is a member of the data safety monitoring board for a COVID-19 vaccine clinical trial sponsored by the Vaccine and Infectious Disease Organization at the University of Saskatchewan; a Leadership Group member, Co-Chair of the Field Studies Working Party and Chair of the Pediatric Network for the Federal COVID-19 Immunity Task Force; and advisor to the Deputy Minister of Health and the Chief Medical Officer of Health as a member of the Alberta Advisory Committee on Immunization. No other competing interests were declared.

This article was solicited and has not been peer reviewed.

Affiliation: Alberta Children's Hospital Research Institute, Cumming School of Medicine, University of Calgary, Calgary, Alta.

Contributors: Stephen Freedman drafted the work. James Kellner revised the manuscript critically for important intellectual content. Both authors contributed to the conception and design of the work, gave final approval of the version to be published and agreed to be accountable for all aspects of the work.
Content licence: This is an Open Access article distributed in accordance with the terms of the Creative Commons Attribution (CC BY-NC-ND 4.0) licence, which permits use, distribution and reproduction in any medium, provided that the original publication is properly cited, the use is noncommercial (i.e., research or educational use), and no modifications or adaptations are made. See: https://creativecommons.org/licenses/by-nc-nd/4.0/

Funding: Stephen Freedman has received grants to conduct SARS CoV-2 research from the following agencies: Health Canada (Safe Restart Agreement Contribution Program), Canadian Institutes of Health Research (CIHR; Emerging COVID-19 Research Gaps and Priorities; Stream 1 of the Operating Grant: SARS-CoV-2 variants supplement: COVID-19 Rapid Research Funding Opportunity; COVID-19 Rapid Research Funding Opportunity - Social Policy and Public Health Response; COVID19 - Clinical management), Public Health Agency of Canada (Immunization Partnership Fund - Emerging Issues Fund Program) and the Alberta Health Service - University of Calgary - Clinical Research Fund. James Kellner has received grants from CIHR, Canadian Immunization Research Network, BC Lung Association, Genome Alberta and Alberta Children's Hospital Foundation, Pfizer, GlaxoSmithKline, Merck and Moderna.

Correspondence to: Stephen Freedman,

Stephen.Freedman@albertahealthservices.ca 